\title{
The Infant With Noisy Breathing
}

\author{
Abby $R$. Nolder, $M D^{1,2}$ \\ Gresham T. Richter, MD, FAAP, FACS ${ }^{1,2, *}$
}

\author{
Address \\ ${ }^{*}, 1$ Department of Otolaryngology Head and Neck Surgery, Division of Pediatric \\ Otolaryngology, University of Arkansas for Medical Sciences, Little Rock, AR \\ 72207, USA \\ Email: GTRichter@uams.edu \\ ${ }^{2}$ Arkansas Children's Hospital, 1 Children's Way, Little Rock, AR 72207, USA
}

Published online: 26 July 2015

C) Springer International Publishing AG 2015

This article is part of the Topical Collection on Otolaryngology

Electronic supplementary material The online version of this article (doi:10.1007/s40746-015-0025-5) contains supplementary material, which is available to authorized users.

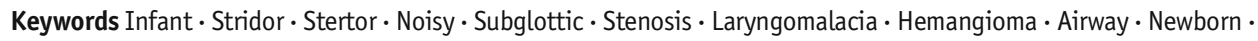
Congenital

\section{Opinion statement}

Evaluation of the infant with noisy breathing can be intimidating as it suggests some degree of airway obstruction. Knowledge of airway anatomy and the common causes of noisy breathing in this patient population is critical in making the diagnosis and determining the next step in clinical management. A focused history with emphasis on birth, intubation, and feeding history as well as a complete head and neck examination often yield the diagnosis. The clinician must be able to differentiate stridor from stertor as well as determine whether the airway noise is inspiratory, expiratory, or biphasic. The phase of respiration in which the noise is best heard is an important clue to the level of airway obstruction. Evaluation with awake flexible fiberoptic laryngoscopy (FFL) and/or sedated airway endoscopy is often required for diagnosis and intervention. Differentiating nasal and pharyngeal sources of obstruction from laryngeal anomalies is important for determining urgency of management. Laryngomalacia, congenital or acquired subglottic stenosis, and airway hemangioma are the most common laryngeal sources of noisy breathing in infants. Respiratory papillomatosis and airway foreign body should also be considered but are less common causes. Proper identification, management, and understanding of the current trends in treating these conditions will ensure the best possible patient outcomes.

\section{Introduction}

Noisy breathing within the first few months of life is a cause for alarm in parents and clinicians alike and is a common reason for presentation to primary care. The goals of the initial evaluation are to determine the most 
likely cause of the noisy breathing and to decide whether consultation with or referral to an otolaryngologist is needed. A quick assessment of the child should be made to determine the severity of the symptoms and whether immediate airway intervention is required. Fortunately, in most situations, this is not the case and a careful history and physical exam can be carried out.

Timing of the onset of noisy breathing should be discussed. Acute onset of noisy breathing often suggests an infectious or inflammatory etiology, while gradual and progressive symptoms indicate a congenital cause. Birth history, including prematurity and whether the child required intubation after birth, is important $[1,2$, $3 \bullet \bullet]$. Prematurity and a history of intubation should raise suspicion for congenital or acquired stenoses, especially in the case of biphasic stridor. One should ask the parent if the infant is having retractions, apneas, or cyanosis and whether the noisy breathing gets better or worse with positioning. Supine positioning often worsens symptoms of laryngomalacia $[3 \bullet \bullet, 4 \bullet \bullet, 5,6]$ but will have very little effect on symptoms secondary to nasal or pharyngeal obstruction. Inquiry into, and mimicking, the sounds of stertor and stridor will improve a parent's ability to provide historic detail on their child's airway problem. Stridor and stertor should also be differentiated from wheezing, which is a lower airway noise. New-onset wheezing in an otherwise healthy child should raise suspicion for airway foreign body, especially in the infant that has started crawling, has older siblings, or has had a witnessed choking event.

A thorough feeding history should also be gathered, as many infants with noisy breathing will also have feeding difficulties due to an uncoordinated suckswallow-breathe sequence. This may result in poor weight gain and failure to thrive and will drive more urgent intervention. Feeding history should include whether the infant is breast or bottle fed; how long it takes the infant to feed; whether the child coughs, chokes, or pulls away from the bottle or breast during the feeding; whether breathing worsens during or after a feeding; and whether the child is having excessive regurgitation or reflux. Observing the child during a feed, in consultation with a speech language pathologist or occupational therapist that specializes in infant feeding, can be helpful. This allows for thoughtful feeding modifications when significant feeding difficulty or recurrent aspiration events are identified. Treatment of symptomatic reflux is also an important factor in managing infants with noisy breathing as extraesophageal reflux can contribute to or exacerbate noisy breathing in many cases, especially in those with laryngomalacia $[3 \bullet \bullet, 7$, 8]. Both feeding difficulties and significant reflux should prompt further evaluation with a video fluoroscopic swallowing study or gastrointestinal work-up.

\section{Physical exam}

Physical examination of the infant with noisy breathing begins with simply observing the child, taking note of the infant's respiratory effort and whether suprasternal or subcostal retractions are present. Airway noise is the result of turbulent airflow vibrating the soft tissues of a narrow

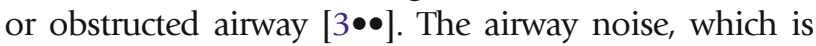
usually audible with or without a stethoscope, should be characterized and will assist in suspecting laryngeal from supralaryngeal and tracheal sources of obstruction (Table 1). However, multilevel airway obstruction is not uncommon in an infant with noisy breathing.

The location of the obstruction is best determined by differentiating stertor from stridor and identifying in which phase of respiration the sound is best heard, i.e., inspiratory, expiratory, or biphasic. Stertor mimics snoring and usually results from obstruction or collapse at the level of

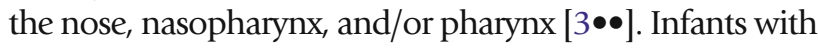
nasal obstruction often present with stertor. This is most likely due to nasal congestion, inferior turbinate hypertrophy, adenoid hypertrophy, a nasolacrimal duct cyst, or pyriform aperture stenosis in decreasing order of frequency. Other potential causes include choanal atresia, glossoptosis, and pharyngomalacia to name a few.

Stridor has a more high-pitched, musical quality and can result from airway obstruction at the level of the supraglottis, glottis, subglottis, or trachea. Inspiratory stridor suggests obstruction of the supraglottis or glottis, as in the case of laryngomalacia and glottis stenosis. Subglottic narrowing from congenital or acquired stenosis, subglottic cysts, or subglottic hemangiomas will typically presents with biphasic stridor. Unilateral vocal cord paresis may cause a weak cry but rarely causes noisy breathing. In contrast, the rare bilateral vocal cord paralysis can be a source of biphasic stridor [9]. Expiratory stridor usually suggests narrowing of the trachea and can most commonly be a result of tracheomalacia or extrinsic tracheal or bronchial compression from a vascular ring $[3 \bullet \bullet$. Complete tracheal rings are an uncommon and dangerous condition associated with severe narrowing of the trachea due to continuous cartilage rings without an intervening trachealis. Infants with this condition more commonly have a washing machine quality of breathing. 
Table 1. Upper airway and tracheal causes of noisy breathing in the infant categorized by sound quality

\begin{tabular}{|c|c|}
\hline Sound quality & Potential etiology \\
\hline \multirow[t]{7}{*}{ Stertor } & Nasolacrimal duct cyst \\
\hline & Pyriform aperture stenosis \\
\hline & Inferior turbinate hypertrophy \\
\hline & Choanal atresia \\
\hline & Adenoid hypertrophy \\
\hline & Pharyngomalacia \\
\hline & Glossoptosis \\
\hline \multirow[t]{4}{*}{ Inspiratory stridor } & Laryngomalacia \\
\hline & Vallecular cyst \\
\hline & Laryngocele \\
\hline & Laryngeal papilloma \\
\hline \multirow{7}{*}{$\begin{array}{l}\text { Inspiratory or biphasic } \\
\text { stridor }\end{array}$} & Subglottic cyst \\
\hline & Bilateral vocal fold paralysis \\
\hline & Subglottic stenosis \\
\hline & Subglottic hemangioma \\
\hline & Glottic stenosis/web \\
\hline & Laryngeal papilloma \\
\hline & Airway foreign body \\
\hline \multirow{6}{*}{$\begin{array}{l}\text { Expiratory stridor/ } \\
\text { grunting }\end{array}$} & Tracheomalacia \\
\hline & Tracheal stenosis \\
\hline & Complete tracheal rings \\
\hline & Mediastinal mass \\
\hline & $\begin{array}{l}\text { Vascular ring-tracheal } \\
\text { compression }\end{array}$ \\
\hline & Airway foreign body \\
\hline
\end{tabular}

A complete head and neck examination should follow the initial assessment to look for any syndromic features or associated lesions such as neck masses or cutaneous hemangiomas that may provide a clue to the etiology of the airway symptoms. A beard distribution of a segmental facial hemangioma should raise high suspicion for subglottic disease. The oral cavity and oropharynx exam should look for cleft palate, tonsil hypertrophy, or lingual hypertrophy secondary to macroglossia or glossoptosis. The nasal exam may indicate congestion or a mass such as a nasolacrimal duct cyst as the cause of stertor and nasal obstruction. Examination of the chest may reveal pectus excavatum, which typically indicates severe upper airway obstruction and likely the result of laryngomalacia, bilateral vocal cord paralysis, or subglottic stenosis $[3 \bullet \bullet, 8$, 10].

\section{Endoscopy}

Flexible fiberoptic laryngoscopy (FFL) is an invaluable tool used frequently by otolaryngologists in evaluating the infant with noisy breathing. With proper equipment and training, this exam can be done safely without sedation and allow for a dynamic evaluation of the airway. Nasal decongestant such as phenylephrine or oxymetazoline is typically used with or without topical anesthetic. A pediatric flexible fiberoptic endoscope is passed atraumatically along the nasal floor to visualize the nasal cavity and nasopharynx, pharynx, supraglottic larynx, and vocal cords. If video recording and playback is available, this is recommended as it often helps to identify subtle lesions that may have been missed on the initial pass of the scope, and provides an opportunity for caregiver education.

Many causes of noisy breathing can be diagnosed with FFL alone, often eliminating the need for operative endoscopy for diagnostic purposes. Subglottic and tracheal lesions may be more difficult to visualize with FFL; however, awake flexible tracheoscopy may be safely used to assess lesions distal to the true vocal folds [11]. Still, some lesions will require further investigation in the operating room under general anesthesia with direct microlaryngoscopy and diagnostic bronchoscopy. Having the patient in the operating room will also allow for operative endoscopic intervention for laryngomalacia, subglottic stenosis, or subglottic hemangioma.

\section{Treatment}

Laryngomalacia (LM) is the most common cause of stridor in infants, occurring in $60-70 \%$ of infants presenting with noisy breathing [4••]. Diagnosis is based on history and physical exam, including FFL that confirms the diagnosis. The hallmark 
findings on flexible laryngoscopy are a tubular or omega-shaped epiglottis with shortened aryepiglottic folds and redundant supra-arytenoid mucosa that prolapses into the airway on inspiration causing airway obstruction (Fig. 1, Video 1). Abnormal neuromuscular tone of the supraglottic larynx is the prevailing theory for the etiology of LM [8]. Infants with LM usually present at the age of 1-6 months with inspiratory stridor since birth or shortly thereafter that worsens with feeding, agitation, or supine positioning. Most infants with LM will also have gastroesophageal reflux (GER) that can exacerbate feeding and airway symptoms. The majority of infants with mild to moderate LM will improve in their airway and feeding issues with GER management alone, with evidence of symptom resolution ranging from 6 to 18 months of age. Most otolaryngologists agree that pharmacologic treatment of clinically significant GER is necessary in the treatment of moderate to severe LM both preoperatively and postoperatively due to the association of GER with laryngeal sensitivity and feeding difficulty $[4 \bullet \bullet, 8,10]$.

Ten to $20 \%$ of children presenting with LM will require surgical intervention for severe airway obstruction resulting in apneas, blue spells, feeding difficulties, and failure to thrive, while others may be treated conservatively with medical management of reflux and close observation $[8,10]$. These patients undergo intraoperative microlaryngoscopy with bronchoscopy and demonstrate evidence of supralaryngeal prolapse upon inspiration (Video 2).

Common secondary airway lesions include grade 1 subglottic stenosis and tracheomalacia [12]. Supraglottoplasty is the accepted procedure for the surgical management of severe LM. These procedures require a general inhalational and intravenous anesthetic with spontaneous ventilation, microlaryngoscopic bilateral release of the shortened aryepiglottic folds, and removal of excess supra-arytenoid tissue (Fig. 1). Carbon dioxide $\left(\mathrm{CO}_{2}\right)$ laser and cold steel instruments have both been used with a success rate of up to $90 \%[4 \bullet \bullet]$; however, outcomes are less favorable in syndromic children and those with medical comorbidities such as neurologic disease $[1,6]$. The risk of complications in skilled hands is very low [1].

While the absolute indications for supraglottoplasty in infants with severe LM are clear (i.e., hypoxia, failure to thrive, pectus excavatum, cyanosis, apnea), relative indications such as feeding difficulty and aspiration without failure to thrive require clinical judgment. Significant improvement in growth curves, GER, and feeding outcomes has been demonstrated after supraglottoplasty $[4 \bullet \bullet, 5,6]$. Therefore, indications for supraglottoplasty could be expanded to include the infant with moderate LM based on airway symptoms but severe GER and dysphagia on clinical exam or video swallow study. A polysomnogram may assist the clinician in determining the severity of disease $[2,3 \bullet \bullet, 4 \bullet \bullet]$. More investigation is needed in the management of moderate laryngomalacia, but evidence suggests that both medical and surgical management improves the quality of life for concerned caregivers $[3 \bullet \bullet, 13 \bullet]$. who presents with biphasic stridor should raise suspicion for a congenital or acquired subglottic stenosis (SGS). While the use of small cuffless or low-pressure cuffed endotracheal tubes has decreased the prevalence of acquired SGS over the last several years, the incidence remains at 1- 

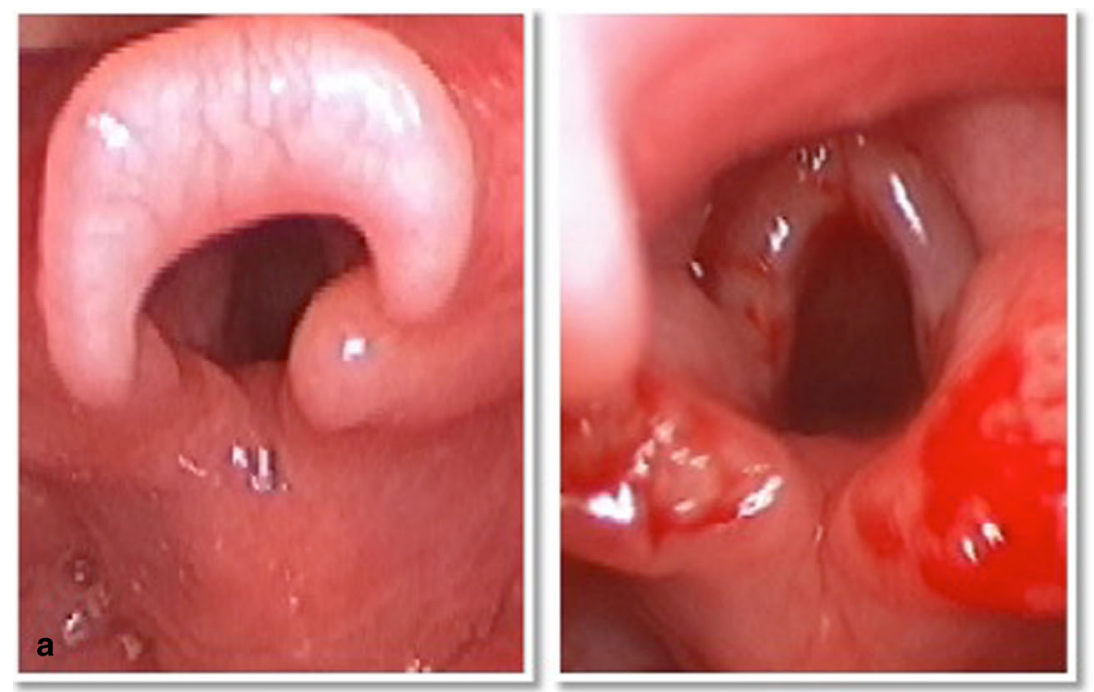

Fig. 1. a Supraglottic view of a 3-monthold infant with severe laryngomalacia before and after intraoperative supraglottoplasty. b Subglottic view of a 6month-old infant with acquired SGS after prolonged intubation for respiratory syncytial virus before and after endoscopic balloon dilation. c Subglottic lumen with a SGH before and after systemic beta-blocker therapy augmented with intralesional steroids.
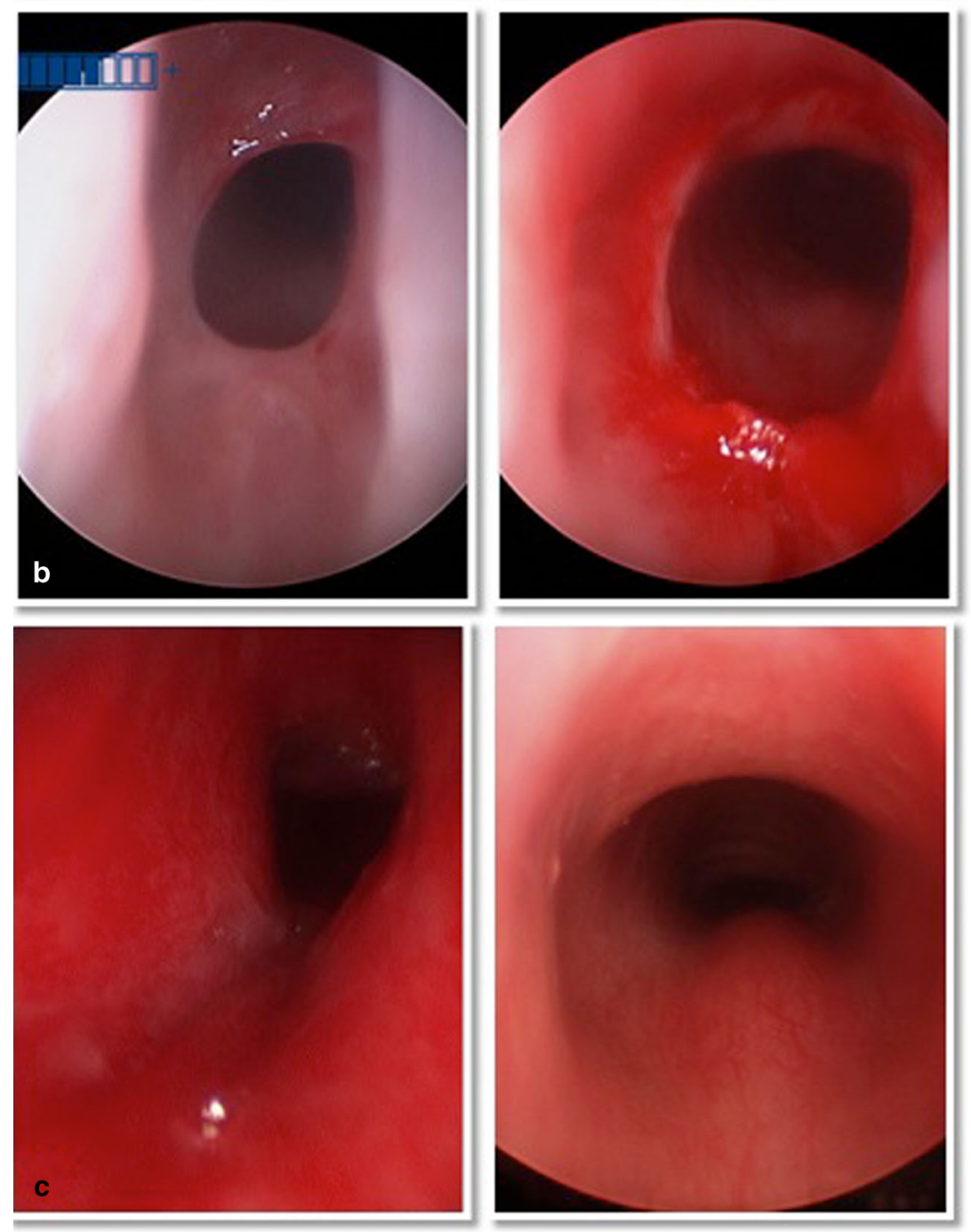
$2 \%$ of intubated neonates $[3 \bullet \bullet, 7,8,14 \bullet \bullet]$. If subglottic stenosis is suspected, the child should be referred to a pediatric otolaryngologist for definitive diagnosis and treatment. Stridor present immediately at birth may indicate a congenital cartilaginous stenosis. In these patients, an elliptical cricoid ring will be visualized endoscopically. An acquired stenosis may be discovered in the stridulous newborn after extubation. This may also delayed a few weeks as a subglottic scar develops (Fig. 1).

Plain film neck X-rays may help with the diagnosis if a steeple sign is seen, but the gold standard for diagnosing SGS is operative airway endoscopy with

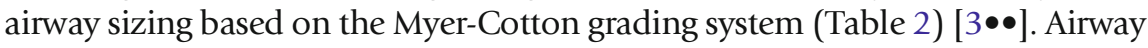
symptoms depend on the grade of stenosis and range from mild biphasic stridor on agitation or feeding with a grade I stenosis to severe respiratory distress requiring tracheotomy for grades II-IV.

Options for treatment also depend on the severity and type of SGS. Congenital lesions are more likely to require open airway reconstruction, while acquired lesions may be better managed initially with balloon dilatation. Congenital lesions demonstrate an elliptical cricoid ring that does not respond to dilation and typically will require augmentation with a cartilage graft to improve the subglottic lumen size.

Endoscopic balloon dilatation laryngoplasty offers the advantage of shorter operative times and decreased length of hospital stay versus open airway procedures, using a less invasive and expedient technique $[3 \bullet \bullet, 15 \bullet]$. A balloon dilator is placed in the airway under direct visual guidance and filled with saline based on manufacturer's recommendations. As the balloon is inflated, it results in radial dilation of the circumferential stenotic scar band without shearing forces $[9,15 \bullet]$. Success is best achieved in early and thin scar bands. Several studies have analyzed the safety, efficacy, and outcomes of balloon dilation compared to and as an adjunct to traditional open airway surgery. Most agree that higher grades of stenosis (grades III-IV), multiple comorbidities, and multilevel airway obstruction lead to less success with endo-

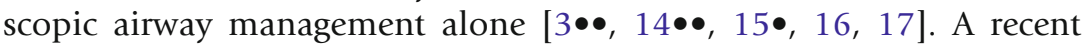
systematic review, however, showed a $64 \%$ overall success rate for balloon dilation $[3 \bullet \bullet, 8,10,14 \bullet \bullet]$ in the management of SGS. Other techniques such as removal of stenotic tissue with a microdebrider or $\mathrm{CO}_{2}$ laser, lysis of scar with cold steel instruments, and the use of topical steroids and antifibroblast agents may be used in conjunction with balloon dilatation to prevent restenosis.

Patients with moderate to severe stenosis (Myer-Cotton grades II-IV) and those who fail endoscopic management may require tracheotomy and/or open airway reconstruction in the form of anterior and/or posterior autologous cartilage graft laryngotracheoplasty (LTP). In infants less than 18 months with

Table 2. Myer-Cotton grading system for subglottic stenosis

$\begin{array}{ll}\text { Grade } & \\ \text { I } & \text { Less than } 50 \% \text { obstruction } \\ \text { II } & 51-70 \% \text { obstruction } \\ \text { III } & 71-99 \% \text { obstruction } \\ \text { IV } & \text { Complete obstruction }\end{array}$


isolated anterior subglottic stenosis, an anterior cricoid split with placement of an interposition graft from the superior (alar) portion of the thyroid cartilage may be sufficient to successfully treat the stenosis and, in many cases, avoid tracheotomy $[11,18,19]$. This is an augmentation of the earlier anterior cricoid split technique used and stabilizes the open cricoid defect with a small amount of cartilage without the morbidity associated with harvesting a costocartilage (rib) graft.

In children older than 18 months and in those requiring both anterior and posterior airway augmentation, rib cartilage is preferred. This procedure may be performed using a single- or double-stage technique. In a single-stage LTP, the tracheotomy is removed at the time of surgery and the patient is intubated with an appropriately sized endotracheal tube. The child remains intubated and sedated in the pediatric intensive care unit for 4-7 days during the healing period. Extubation is performed after airway endoscopy in the operating room to ensure proper healing. The obvious advantage to single-stage LTP is immediate decannulation of the tracheostomy. Disadvantages include the need for prolonged sedation and ventilation, which often results in narcotic habituation and can result in atelectasis or pneumonia $[4 \bullet \bullet, 20]$.

Double-stage LTP is often recommended for patients with more severe stenosis or significant medical comorbidities, such as underlying pulmonary or neurologic disease that may complicate extubation or dependence on a newly reconstructed airway $[8,21]$. In double-stage LTP, the tracheotomy tube is left in place at the time and a Teflon-coated or silastic stent is used to support the cartilage grafts. The stent is then removed 3-4 weeks later. Eventual decannulation of the tracheostomy is considered as the measure of success for double-stage LTP.

Successful decannulation with single- and double-stage LTP ranges from 84 to $96 \%[4 \bullet \bullet, 8,10,22]$. Preoperative assessment and treatment of comorbidities such as GER, eosinophilic esophagitis, and colonization with methicillinresistant Staphylococcus aureus is imperative to successful LTP healing $[8,10,20]$. Endoscopic techniques are still employed to assess healing, remove granulations tissue, or dilate the newly formed airway to stabilize patency.

An alternative to laryngotracheoplasty is the cricotracheal resection (CTR). This technique involves resection of the stenotic segment with primary anastomosis of the trachea to the cricoid plate and may be preferred in high-grade stenosis (Myer-Cotton grades III-IV) that begin several millimeters below the true vocal cords $[12,21]$.

Subglottic hemangiomas (SGHs) are a less common source of noisy breathing in the newborn but an important cause of airway obstruction that requires early attention. SGHs are infantile hemangiomas in the subglottic lumen that arise from the proliferation of vascular endothelial cells. Rapid expansion occurs in the first 6-8 months of life as the tumor forms, leading to progressive inspiratory or biphasic stridor. Cutaneous infantile hemangiomas may indicate the presence of a SGH in the stridulous child, especially in the setting of segmental facial disease. Like other infantile hemangiomas, SGH will enter a slow dissolution (involution) phase that begins around 1 year of life but may last until 47 years. 
Subglottic hemangiomas may partially or completely occlude the newborn airway and cause significant respiratory distress during early growth. Thus, expedient intervention is required. The practice of systemic therapy with an oral non-selective beta-blocker has recently been adopted as a standard therapy $[4 \bullet \bullet, 23,24]$. Routine electrocardiogram is performed to rule out heart block prior to treatment. Propranolol is then administered at a dose of $2-3 \mathrm{mg} / \mathrm{kg} /$ day divided into three-timesa-day dosing until 1 year of age $[1,6,25 \bullet \bullet]$. Blood pressure and heart rate are periodically measured, and caregivers are informed to give the medication with food to circumvent the risk of hypoglycemia. Success rates of propranolol therapy alone for SGH are high, but treatment failures have been reported [26].

Office-based FFL and sedated microlaryngoscopy aid in the diagnosis and management of SGH (Fig. 1). Intralesional steroids administered at the time of microlaryngoscopy will expedite the reduction of large and obstructing SGH. Immediate postoperative intubation of 1 day may be required to allow for edema resolution. A multimodal approach of systematic propranolol in combination with intralesional steroids is very effective (Fig. 1). Prior to propranolol therapy, SGH may have been managed with intralesional steroids, laser ablation, open excision, and LTP or a combination of these procedures. The modality was typically based on the experience of the treating physicians. Repeat procedures are frequently required to control the proliferative phase.

With appropriate endoscopic and medical management, infants with SGH should not require a tracheotomy. However, many infants with a subglottic hemangioma have an associate elliptical cartilage with grade I congenital SGS. If an open procedure is elected to remove the SGH, a thyroid alar laryngotracheoplasty is recommended at the time of surgery to augment the subglottic lumen upon closure.

\section{Conclusion}

Appropriate and timely evaluation is important for the diagnosis and management of infants with noisy breathing. Obstruction from the level of the anterior nose and mouth to the trachea can be differentiated based on sound quality, history, and physical exam. Awake flexible fiberoptic laryngoscopy (FFL) and/or sedated airway endoscopy is often required for diagnosis and intervention. Reflux management and feeding modifications are important in the initial management of many cases. Laryngomalacia, subglottic stenosis, and subglottic hemangioma are common causes of noisy breathing in infants. Laryngomalacia is the most common cause of stridor in the newborn, with surgery reserved for those with airway obstruction, significant feeding difficulties, and/or failure to thrive. Subglottic stenosis should be suspected in preterm infants or term infants with a history of intubation presenting with biphasic stridor. Management depends on the severity of stenosis and includes both endoscopic and open airway reconstruction. Infants with cutaneous hemangiomas, especially those in a beard distribution, presenting with biphasic stridor may have a subglottic disease. The use of 
propranolol has revolutionized the medical management of SGH and is now routinely used, often in conjunction with endoscopic intralesional steroids.

\section{Compliance with Ethics Guidelines}

\section{Conflict of Interest}

Abby R. Nolder and Gresham T. Richter declare that they have no conflict of interest.

Human and Animal Rights and Informed Consent

This article does not contain any studies with human or animal subjects performed by the author.

\section{References and Recommended Reading}

Papers of particular interest, published recently, have been highlighted as:

- Of importance

$\bullet \quad$ Of major importance

1. Preciado D, Zalzal G. A systematic review of supraglottoplasty outcomes. Arch Otolaryngol Head Neck Surg. 2012;138(8):718. doi:10.1001/archoto. 2012.1251.

2. Boudewyns A, Claes J, Van de Heyning P. Clinical practice. Eur J Pediatr. 2009;169(2):135-41. doi:10. 1007/s00431-009-1044-7.

3.• Ida JB, Thompson DM. Pediatric stridor. Otolaryngol Clin N Am. 2014;47(5):795-819. doi:10.1016/j.otc. 2014.06.005.

Provides excellent overview of the evaluation and treatment of the child with stridor.

4.• Dobbie AM, White DR. Laryngomalacia. Pediatr Clin N Am. 2013;60(4):893-902. doi:10.1016/j.pcl.2013.04. 013.

Comprehensive review of laryngomalacia diagnosis and management.

5. Richter GT, Wootten CT, Rutter MJ, Thompson DM. Impact of supraglottoplasty on aspiration in severe laryngomalacia. Ann Otol Rhinol Laryngol. 2009;118(4):259-66.

6. Durvasula VSPB, Lawson BR, Bower CM, Richter GT. Supraglottoplasty outcomes in neurologically affected and syndromic children. JAMA Otolaryngol Head Neck Surg. 2014;140(8):704. doi:10.1001/jamaoto.2014.983.

7. Gnagi SH, Schraff SA. Nasal obstruction in newborns. Pediatr Clin North Am. 2013;60(4):903-22. doi:10. 1016/j.pcl.2013.04.007.

8. Thompson DM. Laryngomalacia: factors that influence disease severity and outcomes of management. Curr Opin Otolaryngol Head Neck Surg. 2010;18(6):564-70. doi:10.1097/MOO. ob013e3283405e48.
9. Chen EY, Inglis Jr AF. Bilateral vocal cord paralysis in children. Otolaryngol Clin N Am. 2008;41(5):889901. doi:10.1016/j.otc.2008.04.003.

10. Richter GT, Thompson DM. The surgical management of laryngomalacia. Otolaryngol Clin N Am. 2008;41(5):837-64. doi:10.1016/j.otc.2008.04. 011.

11. Hartzell LD, Richter GT, Glade RS, Bower CM. Accuracy and safety of tracheoscopy for infants in a tertiary care clinic. Arch Otolaryngol Head Neck Surg. 2010;136(1):66-9. doi:10.1001/archoto. 2009.204.

12. Dickson JM, Richter GT, Meinzen-Derr J, Rutter MJ, Thompson DM. Secondary airway lesions in infants with laryngomalacia. Ann Otol Rhinol Laryngol. 2009;118(1):37-43.

13. Kilpatrick LA, Boyette JR, Hartzell LD, et al. Prospective quality of life assessment in congenital laryngomalacia. Int J Pediatr Otorhinolaryngol. 2014;78(4):583-7. doi:10.1016/j.ijporl.2014.01.001.

Addresses caregiver impact of laryngomalacia.

14.• Wentzel JL, Ahmad SM, Discolo CM, Gillespie MB, Dobbie AM, White DR. Balloon laryngoplasty for pediatric laryngeal stenosis: case series and systematic review. Laryngoscope. 2014;124(7):1707-12. doi:10. 1002/lary.24524.

Systematic review of the use of endoscopic balloon dilation of subglottic stenosis.

15. Maresh A, Preciado DA, O'Connell AP, Zalzal GH. A comparative analysis of open surgery vs endoscopic balloon dilation for pediatric subglottic stenosis. JAMA Otolaryngol Head Neck Surg. 2014;140(10):901. doi:10.1001/jamaoto.2014.1742. 
Excellent comparison of open and endoscopic techniques in the management of SGS.

16. Bakthavachalam S, McClay J. Endoscopic management of subglottic stenosis. Otolaryngol Head Neck Surg. 2008;139(4):551-9. doi:10.1016/j.otohns.2008.07.024.

17. Quesnel AM, Lee GS, Nuss RC, Volk MS, Jones DT, Rahbar R. International journal of pediatric otorhinolaryngology. Int J Pediatr

Otorhinolaryngol. 2011;75(5):652-6. doi:10.

1016/j.ijporl.2011.02.002.

18. Schroeder Jr JW, Holinger LD. Congenital laryngeal stenosis. Otolaryngol Clin N Am. 2008;41(5):865-75. doi:10.1016/j.otc.2008.04.015.

19. Nguyen CV, Bent JP, Shah MB, Parikh SR. Pediatric primary anterior laryngotracheoplasty: thyroid ala vs costal cartilage grafts. Arch Otolaryngol Head Neck Surg. 2010;136(2):171-4. doi:10.1001/archoto.2009.224.

20. Meier JD, White DR. Multisystem disease and pediatric laryngotracheal reconstruction. Otolaryngologic Clinics of NA. 2012;45(3):643-51. doi:10.1016/j.otc. 2012.03.004.

21. Bajaj Y, Cochrane LA, Jephson CG, et al. International journal of pediatric otorhinolaryngology. Int J Pediatr Otorhinolaryngol. 2012;76(4):507-11. doi:10.1016/j. ijporl.2012.01.006.
22. Schmidt RJ, Shah G, Sobin L, Reilly JS. International journal of pediatric otorhinolaryngology. Int J Pediatr Otorhinolaryngol. 2011;75(12):1585-8. doi:10.1016/ j.ijporl.2011.09.012.

23. Anderson de Moreno LC, Matt BH, Montgomery G, Kim Y-J. Propranolol in the treatment of upper airway hemangiomas. Ear Nose Throat J. 2013;92(4-5):20914.

24. Buckmiller LM, Munson PD, Dyamenahalli U, Dai Y, Richter GT. Propranolol for infantile hemangiomas: early experience at a tertiary vascular anomalies center. Laryngoscope. 2010;120(4):676-81. doi:10.1002/lary. 20807.

25.• Drolet BA, Frommelt PC, Chamlin SL, et al. Initiation and use of propranolol for infantile hemangioma: report of a consensus conference. Pediatrics. 2013;131(1):128-40. doi:10.1542/peds. 2012-1691.

Multidisciplinary guideline for the initiation and use of propranolol in the management of hemangioma.

26. Goswamy J, Rothera MP, Bruce IA. Failure of propranolol in the treatment of childhood haemangiomas of the head and neck. Laryngol Otol. 2011;125(11):1164-72. 